\title{
DISTRIBUCIÓN DEL PATRÓN ESPACIAL TIPO LEOPARDO EN REGIONES ÁRIDAS Y SEMIÁRIDAS DEL MUNDO
}

\author{
José Fuentes Delgado ${ }^{1}$ \\ Diego Varga Linde ${ }^{2,3}$ \\ Martí Boada Junca ${ }^{1,3}$ \\ ${ }^{1}$ Departamento de Geografía. Universidad Autónoma de Barcelona \\ ${ }^{2}$ Departamento de Geografía. Universidad de Girona \\ ${ }^{3}$ Instituto de Ciencia y Tecnología Ambiental. Universidad Autónoma de Barcelona \\ mcfuentes2@hotmail.com, diego.varga@udg.edu, diego.varga@uab.cat, marti.boada@uab.cat
}

\section{RESUMEN}

Este artículo analiza la generación de un mapa de distribución potencial del patrón espacial leopardo y del entorno asociado a su desarrollo en las zonas áridas y semiáridas. A partir de Información bibliográfica alrededor del mundo y de datos de campo obtenidos en Colombia y España, para ello se caracterizan los factores utilizando predictores ambientales globales en conjunto con un modelo de máxima entropía. En el modelo de distribución, las condiciones ambientales indican que la aridez y la variabilidad topográfica, son factores de peso que tiene una gran valor en las estadísticas que definen los patrones, revelando una extensa distribución de ambientes distintivos nuevos al alrededor del mundo, así como el fenómeno asociado de convergencia evolutiva y de algunos géneros vegetales predominantes en varios continentes a los que se puede asociar este patrón.

Palabras clave: Patrón leopardo, Maxent, Convergencia evolutiva, Zonas áridas.

\section{ABSTRACT}

This paper analyzes the creation of the potential distribution map of the spatial leopard pattern and the environment in relationship to its development in arid and semiarid areas.

Fecha de recepción: mayo 2014.

Fecha de aceptación: abril 2015. 
The information was base on bibliographic information from places around the world and field data obtained in Colombia and Spain. The factors are characterized using global environmental predictors in conjunction with a maximum entropy model. In the model of distribution, the environmental conditions indicate that the aridity and topographic variability are significant factors that have a great influence on the statistics that define patterns, thus revealing a widespread distribution of new distinctive environments around the world, as well as the associate convergent evolution and some dominant plant genera in several continents to which you can associate this pattern.

Keywords: Leopard pattern, Maxent, Convergent evolution, Drylands.

\section{INTRODUCCIÓN}

En las áreas áridas y semi áridas del mundo (Meigg, 1953) durante los últimos 50 años, se ha evidenciado la presencia de patrones espaciales a escala regional y local, en el que la vegetación forma patrones regulares (Slatyer, 1959; Warren, 1973; Worral, 1959), estos pueden ser observados principalmente desde sensores remotos montados sobre plataformas satelitales o aerotransportadas. El avance en las tecnologías de percepción remota significa que el mapeo y monitoreo mediante imágenes de satélite es ahora factible y rentable (White, 2011). Con el constante y mayor aumento de la cobertura y resolución espacial de la información cartográfica, se ha podido vislumbrar con más detalle a un nivel global la presencia de estos comportamientos en la distribución de la vegetación, los patrones consisten principalmente en una matriz heterogenia de cobertura vegetal baja que aparentemente ha sido desestabilizada con la consecuente ruptura del sistema matricial, ésta cubierta vegetal subsecuente revela zonas de suelos desnudos o claros de tierra y zonas con vegetación espaciada de manera regular, generalmente de porte bajo o intercala con porte un poco más alto de vegetación, las áreas son homogéneas en su composición desde el punto de vista de suelos, vegetación y clima circundante. Dentro de la zonas con vegetación se puede presumir una mayor capacidad de desarrollo de las planta por el fenómeno de isla de fertilidad; isla fértil o parches fértiles (Schlesinger, 1990; García, 1970) así como la influencia de la teoría de islas; respecto al equilibrio y dinamismo (MacArthur, 1967) de las especies vegetales presentes en los parches, en el cual los lugares donde se sitúa la vegetación tienen una mayor cantidad de nutrientes dentro del suelo y disponibilidad de humedad, facilitando el desarrollo en estos espacios en contra posición a los lugares donde hay ausencia de la vegetación. En algunos ecosistemas, los parches densos forman bandas o rayas y las comunidades que presentan este patrón se denominan genéricamente como bandas o «patrón tigre». En otros ecosistemas, los parches de vegetación densa son irregulares en forma siguiendo la analogía anterior, aquí se conocen como manchas de vegetación o «patrón leopardo» (Aguiar, 1999). Los patrones espaciales en la vegetación de zonas áridas y semi áridas, se produce en forma de rayas (arbusto tigre - Tiger bush), laberintos, puntos (arbusto leopardo - Leopard bush) y lagunas (Rietkerk, 2008). Se caracterizan por un espaciamiento regular entre los parches de vegetación y pueden abarcar cientos o miles de kilómetros cuadrados de zonas áridas o desérticas (Figura 1). 
La mayoría de los estudios en la bibliografía se han centrado en el caso de las franjas o rayas conocidas como Tiger Bush por su nombre en inglés, muy pocos han sido los estudios que abordan el patrón de manchas o espacios de vegetación denominado leopardo, gran parte de los análisis de patrones espacio-temporales ha sido a través de simulaciones numéricas (Borgogno, 2009), existe un modelo de distribución mundial de patrones periódicos de la vegetación en zonas áridas y semi áridas en el cual intentan aislar la causalidad de todos los patrones en conjunto no haciendo distinción entre ellos y a una escala global poco detallada (Deblauwe, 2008). A causa de la poca investigación y análisis experimentales en este campo, todavía se desconocen los procesos que controlan la formación y el desarrollo especifica del patrón leopardo. Existente diversas hipótesis pero la mayoría señalan que la formación de éstos patrones de vegetación está vinculada al pastoreo de los herbívoros, el fuego, el viento, las condiciones anisotrópicas ambientales en particular la pendiente y otros factores como la diseminación de corta y larga distancia, además de la competitividad entre especies de la vegetación (Chenget, et al., 2010), ligado con el fenómeno de la facilitación, el cual puede llegar a ser factor dominante en la regulación del sistema.

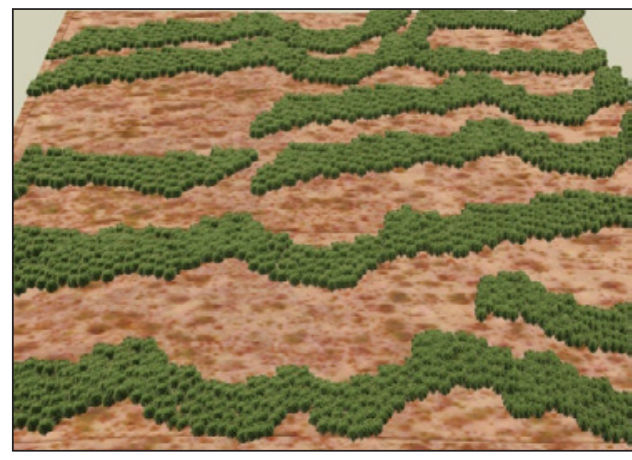

Vista aérea del patrón en bandas o rayas (Tigre)

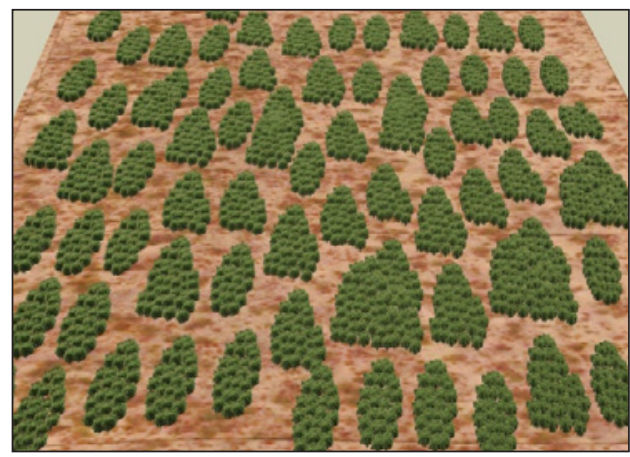

Vista aérea del patrón en espacios (Leopardo)

Fuente: elaboración propia.

Se determinó las relaciones directas de variables ambientales con la aparición y comportamiento específicamente de patrones tipo leopardo en las zonas áridas y semi áridas, tomando como base información real de campo de dos partes del mundo y referencias de información bibliográfica así como predictores de variables ambientales a escala global, en conjunto con un modelo matemático empírico. Con el objetivo de aislar las condiciones ambientales que determinan su existencia en diversas zonas áridas, partiendo de la hipótesis de que en los distintos sitios del mundo dichas variables serán similares; por último contrastando los sitios resultantes con imágenes de alta resolución espacial para verificar los resultados. 


\section{METODOLOGÍA}

\section{II.1. Muestra de patrones (Presencia)}

Los puntos de presencia de patrones leopardo alrededor del mundo fueron recopilados a partir de información bibliográfica (Tongway 1990; Slatyer 1959; Aguiar 1999; Bisigato, 2009; Juergens, 2012; Yetemen, 2010; Bestelmeye, 2006; Borgogno, 2009; Wickens 1971; Lefever, 2009; Meron, 2004) (Tabla 1) y de datos recolectados en campo (Tabla 2) en áreas

Tabla 1

DATOS DE PRESENCIA DEL PATRÓN LEOPARDO TOMADOS DE REFERENCIAS BIBLIOGRÁFICAS

\begin{tabular}{|c|c|c|c|c|c|}
\hline Lugar & Continente & Distancia & Especie & Referencia & Precipitación \\
\hline $\begin{array}{l}\text { Sturk national } \\
\text { Park }\end{array}$ & Australia & $10-50 \mathrm{~m}$ & $\begin{array}{l}\text { Acacia anuera } \\
\text { Eragrostis eriopoda savanna } \\
\text { Monachather paradoxa savanna }\end{array}$ & Tongway 1990 & $308 \mathrm{~mm}$ \\
\hline Alice Springs & Australia & $20-40 m$ & $\begin{array}{l}\text { Acacia anuera } \\
\text { Eragrostis eriopoda savanna }\end{array}$ & Slatyer 1959 & $250 \mathrm{~mm}$ \\
\hline $\begin{array}{l}\text { Río Mayo - } \\
\text { Argentina }\end{array}$ & América & $2 \mathrm{~m}$ & \begin{tabular}{|l|} 
Stipa speciosa \\
Swietenia humilis \\
Poa ligularis \\
Mulinum spinosum \\
Adesmia campestris \\
Senecio filaginoides
\end{tabular} & Aguiar 1999 & $160 \mathrm{~mm}$ \\
\hline $\begin{array}{l}\text { Desierto } \\
\text { del Monte, } \\
\text { Argentina }\end{array}$ & América & $2-3 m$ & $\begin{array}{l}\text { Prosopis flexuosa } \\
\text { Larrea divaricata } \\
\text { Aristida adscensionis } \\
\end{array}$ & Bisigato, 2009 & $30-350 \mathrm{~mm}$ \\
\hline \begin{tabular}{l|} 
Sevilleta \\
WildlifeNational \\
Refuge New \\
Mexico
\end{tabular} & América & $20-40 m$ & \begin{tabular}{|l|} 
Juniperus monosperma \\
Bouteloua eriopoda \\
Larrera tridentata \\
Erioneuron pulchellum \\
\end{tabular} & Yetemen, 2010 & $250 \mathrm{~mm}$ \\
\hline $\begin{array}{l}\text { Chihuahua Las } \\
\text { Cruces, NM }\end{array}$ & América & $1-2 m$ & $\begin{array}{l}\text { Pleuraphis mutica } \\
\text { Prosopis glandulosa Torr } \\
\text { Flourensia cernua } \\
\text { Lycium berlandieri } \\
\text { Larrea tridentata } \\
\end{array}$ & Bestelmeye, 2006 & $220 \mathrm{~mm}$ \\
\hline Zambia & África & $15-30 \mathrm{~m}$ & No hay datos & Borgogno, 2009 & $230 \mathrm{~mm}$ \\
\hline $\begin{array}{l}\text { Kordofán y } \\
\text { Darfur - Sudan }\end{array}$ & África & $30-50 m$ & $\begin{array}{l}\text { Acacia mellifera } \\
\text { Terminalia brownii }\end{array}$ & Wickens 1971 & $350 \mathrm{~mm}$ \\
\hline $\begin{array}{l}\text { South- } \\
\text { WestNiger }\end{array}$ & Africa & $1.75-50 \mathrm{~m}$ & Combretum micranthum & Lefever, 2009 & $200 \mathrm{~mm}$ \\
\hline Namibia & África & $3-5 m$ & \begin{tabular}{|l|} 
Stipagrostis sabulicolae \\
Cladoraphis spinosus
\end{tabular} & Juergens, 2012 & $285 \mathrm{~mm}$ \\
\hline Negev - Israel & Asia & $1-2 m$ & $\begin{array}{l}\text { Paspalum vaginatum } \\
\text { Poa bulbosa }\end{array}$ & Meron, 2004 & $200 \mathrm{~mm}$ \\
\hline
\end{tabular}

Fuente: elaboración propia. 
no antes reportadas como es el caso, en la península de la Guajira en Colombia, las áreas de Almería y Murcia en España (Figura, 2), los puntos de referencia de la información bibliografía fueron verificados utilizando imágenes de alta resolución disponibles en los servicios en el línea BingMaps y Google Earth, estos sistemas incluyen cientos de terabytes en imágenes aéreas y de satélite. En muchas áreas, la resolución máxima es de menos de un metro. En otros lugares, especialmente en las zonas más remotas del mundo, resolución es magnitud menor a 5 metros. Luego de verificar todas las localidades se determinaron 150 puntos de presencia del patrón Leopardo alrededor del mundo (Figura 3).

Tabla 2

DATOS DE PRESENCIA TOMADOS EN CAMPO DEL PATRÓN LEOPARDO

\begin{tabular}{|l|l|l|l|l|}
\hline Lugar & Continente & Distancia & Especie & Precipitación \\
\hline $\begin{array}{l}\text { La Guajira, } \\
\text { Colombia }\end{array}$ & América & $10-50 \mathrm{~m}$ & $\begin{array}{l}\text { Castela } \text { erecta } \\
\text { Heterostachys ritteriana } \\
\text { Prosopis juliflora }\end{array}$ & $152-308 \mathrm{~mm}$ \\
\hline $\begin{array}{l}\text { Almería y Murcia, } \\
\text { España }\end{array}$ & Europa & $2-5 \mathrm{~m}$ & $\begin{array}{l}\text { Acacia anuera } \\
\text { Eragrostis eriopoda savanna } \\
\text { Monachather paradoxa savanna } \\
\text { Arthrocnemum fruticosum } \\
\text { Launaea lanifera } \\
\text { Lycium intricatum } \\
\text { Salsola genistoides } \\
\text { Salsola } \text { oppositifolia }\end{array}$ & $199-289 \mathrm{~mm}$ \\
\hline
\end{tabular}

Fuente: elaboración propia.

Figura 2

PATRONES ESPACIALES TIPO LEOPARDO (IZQUIERDA) LA GUAJIRA, COLOMBIA 2013

$\left(12^{\circ} 9^{\prime} 36.17^{\prime \prime} \mathrm{N}-72^{\circ} 7^{\prime} 44.64^{\prime \prime} \mathrm{)}\right)$ (DERECHA) ALMERIA, ESPAÑA 2010 (3652'52.26"N - 2²0'39.02"O)
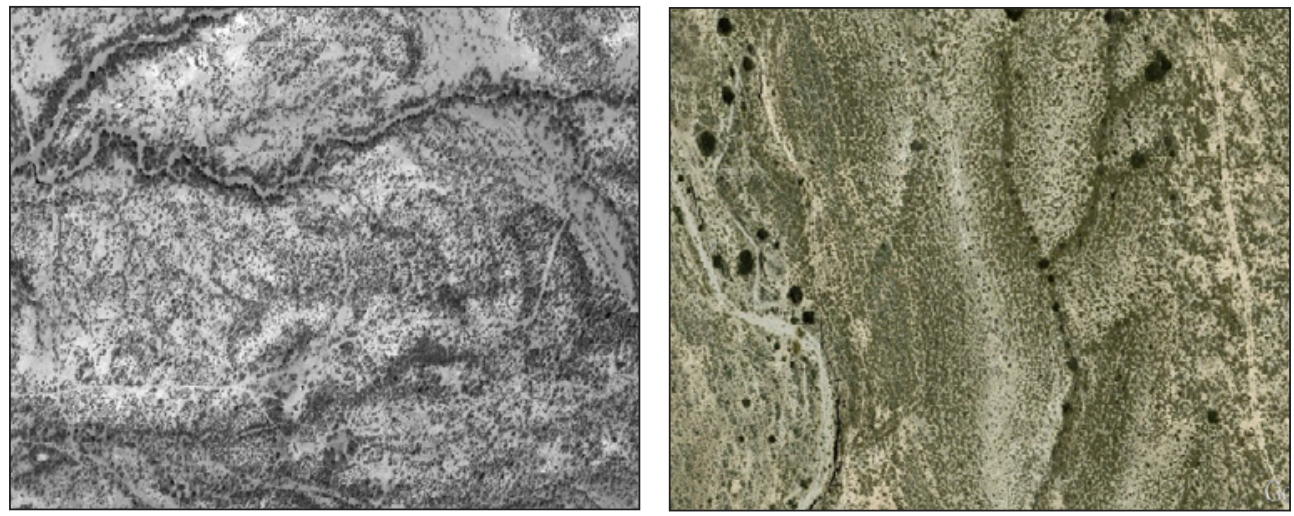

Fuente: EROS-B Imagesat internacional, Ortofoto PNOA, IGN. 


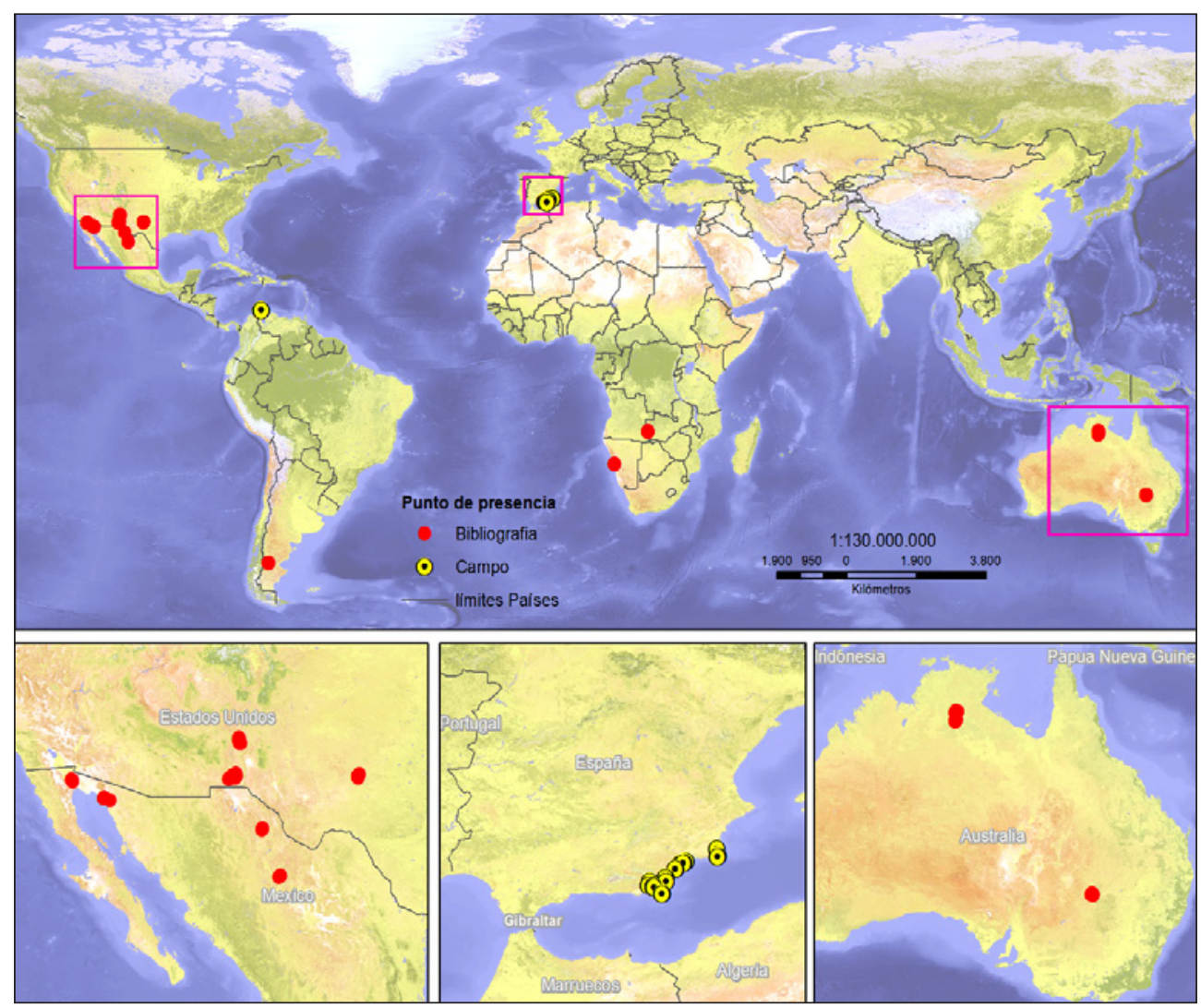

Fuente: elaboración propia.

\section{II.2. Variables ambientales y datos climáticos}

Las variables ambientales refinaron la aplicación de la técnica de modelación en conjunto con un modelo de máxima entropía, se trabajó con un set de variables bioclimáticas predefinidas para caracterizar del espacio ambiental donde se distribuyen las variables climáticas, topográficas y edafológicas. Para evitar que el modelo de distribución tuviera un exceso de información y minimizar el número de variables ambientales correlacionadas, elegimos un subconjunto donde todos los coeficientes de correlación fueron menos de 0,80 . La resolución de los datos fue de $0,5^{\circ}$ para todas las capas de análisis disponibles se consideraron 6 capas temáticas relevantes como predictores del modelo. La temperatura y precipitación anual, fueron obtenidas de WorldClim (http://WorldClim. org) dentro de su base de datos climática global para fines de modelamiento ambiental (1950-2000) (Hijmans, 2005), El índice de aridez global y la radiación solar extraterrestre 
están basados en el modelado y análisis por Antonio Trabucco, (se ofrecen en línea por el CGIAR-CSI con el apoyo del Centro Internacional de Agricultura Tropical CIAT son a la vez el modelo, utilizando los datos disponibles de WorldClim). La elevación fue basada en los datos Shuttle Radar Topography Mission (por sus siglas en ingles SRTM) completos para el mundo en la versión 4, disponible en el CGIAR-CSI SRTM de 90m base de datos derivada (Jarvis, 2008). Finalmente se utilizó base de datos armonizada mundial de los suelos Harmonized World Soil Database (HWSD) (Nachtergaele, 2012) todas las capas fueron cortadas en la misma referencia espacial y tamaño de pixel para poder ser procesadas en formato raster.

\section{II.3. Modelo de distribución potencial}

En los últimos años ha tomado fuerza el uso de técnicas basadas en algoritmos específicos como máxima entropía y algoritmos genéticos (Phillips, 2006; Stockwell, 1992 y 1999). Han significado un aumento exponencial en el número de publicaciones y estudios asociados a la modelación de la distribución geográfica de las especies y ecosistemas, por la facilidad de su aplicación y la rapidez con que se pueden obtener resultados (Pliscoff, 2011). Para generar el modelo empírico potencial se utilizó Maxent (Version 3.3.3) un algoritmo de máxima entropía (Phillips, 2006; Elith, 2011), este muestra la probabilidad de que una celda dada, se tenga una muestra de presencia, para ello utiliza un índice de similitud dentro de unas variables ambientales (predictores) respecto a las celdas donde se ha observado a la especie. Los valores de dicho índice tendrán valores de probabilidad más altos en los espacios semejantes a los lugares donde se ubicaron las muestras conocidas. El modelo inicial permitió generar un mapa de distribución potencial de presencia del patrón Leopardo a nivel global basado en las características de los lugares reportados en campo y en la bibliografía.

Los Modelos de distribución de especies (por sus siglas en ingles SDM) estiman la relación entre registros de especies en los sitios y el medio ambiente y / o características espaciales de esos sitios (Franklin, 2009). Maxent es un método de aprendizaje computacional de propósito general con una simple y la formulación matemática precisa y tiene una serie de aspectos que lo hacen muy adecuado para el modelado de la distribución de especies (Phillips et al., 2006). Es un método no paramétrico, el cual genera una distribución de probabilidad sobre los píxeles de la grilla, empieza por una distribución uniforme y de forma iterativa mejora el ajuste de la distribución a los datos de muestreo; para ello tiene en cuenta las curvas de respuesta, los puntos de muestreo para realizar el test del modelo son una muestra tomada al azar de las localidades de presencia. Maxent utiliza el mismo conjunto de datos tomados al azar en cada vuelta del modelo. Al final del proceso, el valor de la ganancia indica cómo esta de ajustado el modelo a las muestras de presencia (Phillips, 2005). Los valores de salida de las distribuciones predichas son acumulativos, con el valor de salida de una celda de la cuadrícula correspondiente a la suma de las probabilidades de todas las cuadrículas de menor o igual valor a la probabilidad de la celda considerada. En otras palabras, el conjunto de valores más altos que $5 \%$ incorpora el $95 \%$ de la distribución de probabilidad alrededor de la óptima ecológica (Deblauwe, 2008). 


\section{II.4. Evaluación del rendimiento general del modelo}

Se evaluó el rendimiento general del modelo mediante el cálculo del área bajo la curva (AUC), para lo cual se utilizó el 25\% de los puntos de muestra para ser excluidos de los registros y no usarlos en la prueba. La curva ROC es un indicador de la predicción de una acertada presencia o ausencia en la distribución de los datos dentro de los umbrales. Se analizan los valores de las medidas de umbrales independientes, uno para entrenar y el otro para testar el modelo, lo normal es que la curva AUC de entrenamiento aparezca por encima de la curva AUC de test. La curva de entrenamiento representa el ajuste del modelo a los datos de muestreo. La curva test indica el grado de ajuste del modelo a los datos de test, y supone el test real del poder predictivo del modelo (Phillips, 2005). Los resultados sobre la predicción del AUC pueden variar de 0 a 1 , lo cual indica una que la predicción es aleatoria cuando es próximo a 0.5 y una muy buena predicción cuando el umbral se acerca a 1 . Dentro del estudio se tuvo en cuenta las imitaciones conocidas del AUC, las cuales se refieren a la variación del valor de puntuación más alto, cuando la zona de muestreo es inferior en extensión a la distribución de las muestras. Los datos de distribución de especies se refiere a un ámbito geográfico concreto y el aumento de la extensión geográfica presente fuera del dominio del medio ambiente implica la obtención de las puntuaciones más altas de las AUC (Lobo, 2008). Teniendo en cuenta las limitaciones del AUC, el uso de este puede proporcionar información valiosa al utilizar en lugares relativamente homogéneos y con muestras definidas e iguales escalas como es nuestro caso.

\section{RESULTADOS}

El resultado mostro la distribución probable de las áreas en el globo donde se puede evidenciar patrones regulares tipo Leopardo, dentro de las contribuciones relativas de las variables ambientales en el modelo de Maxent, el predictor que tuvo una mayor aporte porcentual fue el índice de aridez global, el que tuvo un 40.2, seguido del predictor de elevación global STRM con un 18.3 éstas dos variables representan más del $50 \%$ de la contribución total a los valores más significativos del índice. La precipitación 14.5, la temperatura 12.4 y en menor medida la radiación solar con 9.8 y los suelos con 4.9. Para el modelo ejecutado las puntuaciones de las AUC fueron estadísticamente muy significativa 0.993, dándole un gran valor predictivo a el modelo de distribución resultante, al superar el límite medio de 0.5 con una mayor aproximación al valor máximo 1, lo que confirmó la pertinencia de ésta predicción al revelar zonas nuevas del mundo donde no se han tenido reportes de presencia del patrón leopardo anteriormente (Figura 4).

El modelo revelo la extensa distribución de ambientes distintivos para la presencia del patrón leopardo, arrojo zonas no identificadas anteriormente o con muy pocas referencias. Estas áreas incluyen el desierto de Chihuaha y Sonora entre Estados unidos y México (Figuras 4a), el sur de Ecuador e islas Galápagos y el norte del Perú en el área de Piura (Figura 4b), una franja costera desde de Libia hasta Gambia, en África Occidental alrededor del desierto del Sahara (Figura 4c), Sudáfrica y desde Angola hasta Namibia por la franja costera alrededor del desierto del Kalahari (Figuras 4d). Áreas alrededor de Somalia, Kenia Yemen y Oman (Figura 4e) y finalmente áreas al borde del desierto australiano 
(Figura 4f). Otras características ambientales importantes que se definieron en las áreas aisladas fueron: la precipitación anual, que se encuentra el rango de $100 \mathrm{~mm}$ a $300 \mathrm{~mm}$, el rango temperatura que es característicamente muy importante en ambientes áridos y en donde los patrones ocurren dentro de la gama de 10 a $26^{\circ} \mathrm{C}$, la elevación que muestra un rango entre 0 a los 1700 metros, la radiación solar que se encuentra en los rango de 10 a $15 \mathrm{~mm} / \mathrm{mes}$ y por último el índice de aridez que se encuentra en el rango de 0 a 0.2 dentro de la característica de árido, en la clasificación de las zonas áridas de la UNESCO (De Pauw, 2000). Referente a las propiedades del suelo predominan los calcisoles suelos con orígenes calcáreos muy típicos de ambientes áridos, donde el material original lo constituyen depósitos aluviales, coluviales o eólicos de materiales alterados.

Figura 4

MODELO DE DISTRIBUCIÓN POTENCIAL DEL PATRÓN ESPACIAL TIPO LEOPARDO
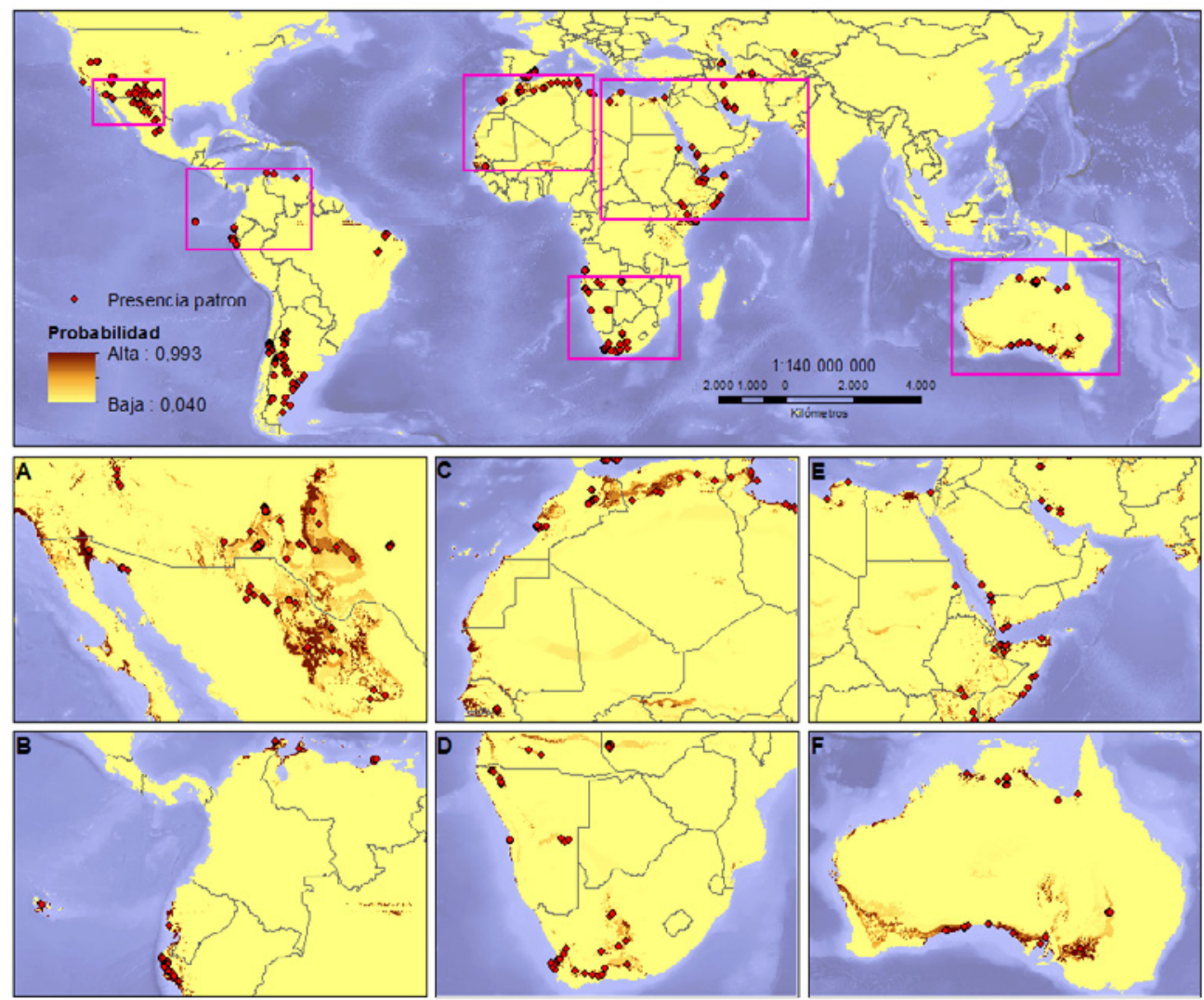

Fuente: elaboración propia.

Se identificaron lugares con ambientes distintivos, con una clara semejanza entre sí, una de estas características se comprobó en los trabajos de campo llevados a cabo en 
España y Colombia. Donde se encontró evidencia del fenómeno llamado convergencia evolutiva (Arendt, 2008), en estos lugares los patrones leopardo se conforman principalmente por dos especies Castela erecta y Lycium intricatum (Figura 5), estas plantas pertenecientes a familias distintas Simaroubaceae y Solanaceae; son muy parecidas en su aspecto feno-morfológico y estructural, pero sin una conexión biológica y evolutiva directa, conformando patrones afines de desarrollo en el ambiente árido donde se desenvuelven, siendo probable que sean modificadas de manera similar y sujetas a presiones de selección similares.

Figura 5

ESPECIES IDENTIFICADAS EN CAMPO (IZQUIERDA) GUAJIRA, COLOMBIA

$\left(12^{\circ} 24^{\prime} 31.34^{\prime \prime} \mathrm{N}-71^{\circ} 43^{\prime} 59.63^{\prime \prime} \mathrm{O}\right)$ (DERECHA) MURCIA, ESPAÑA $\left(37^{\circ} 36^{\prime \prime} 18.62^{\prime \prime} \mathrm{N}-0^{\circ} 43^{\prime} 43.55^{\prime \prime} \mathrm{O}\right)$

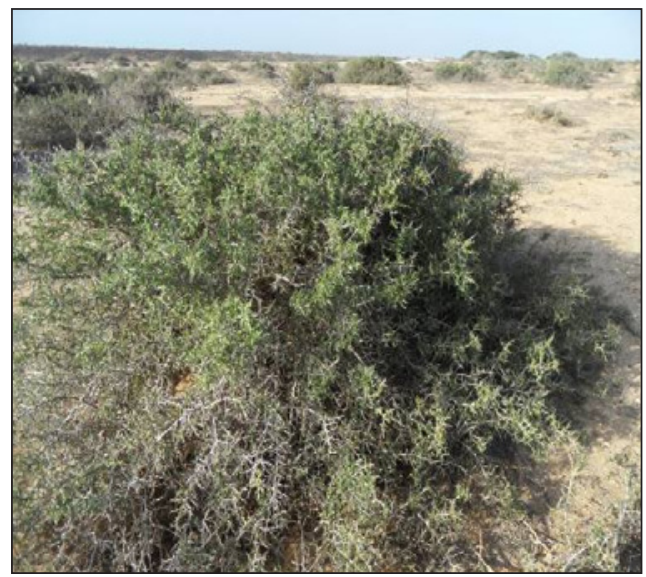

Castela erecta - Simaroubaceae

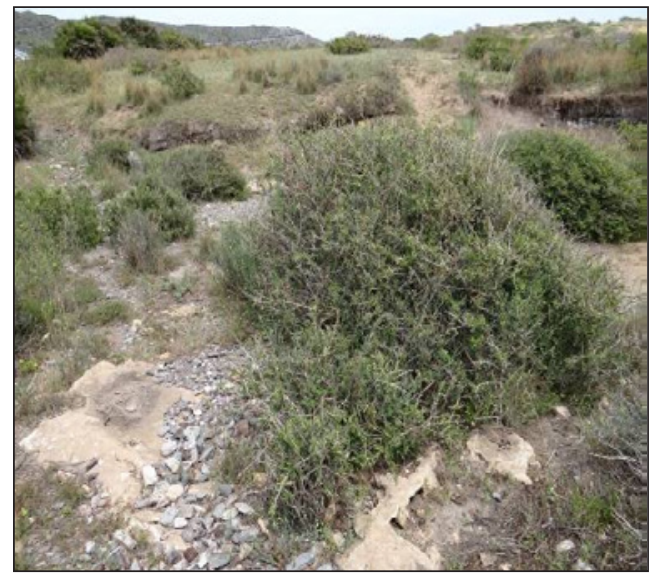

Lycium intricatum - Solanaceae

Para comprobar la estimación de la exactitud de la clasificación, se tomaron 100 puntos de muestra seleccionados sobre el modelo de distribución potencial, se utilizó la matriz de confusión, para ello se seleccionaron los puntos sobre los valores superiores al 0.90. El objetivo es evidenciar la proporción de muestras correctamente clasificadas, comprobando si los patrones se encuentran presentes a través de las imágenes de satélite disponibles en línea utilizando los servicios cartográficos de BingMaps y Google Earth. Generalmente es admisible para una muestra la relación entre tamaño del universo, un error estándar relativo del 10 por ciento. Cuando la matriz de confusión presenta muchas categorías se requiere de un gran número de muestras para evaluar su exactitud, pero en este caso solo se utiliza una única clase. El resultado fue de una precisión con una fiabilidad global del $91 \%$ esto se comprobó con las muestras cotejadas en distintos lugares del mundo donde se verifico la existencia del patrón leopardo, en muchos de los lugares donde no se tenía reportes anteriores o no existían referencias directas debido a la amplia distribución global (Figura 6). 
Figura 6

MUESTRAS DE ALGUNOS LUGARES CON PRESENCIA DEL PATRÓN LEOPARDO ALREDEDOR DEL MUNDO, IDENTIFICADOS CON EL MODELO DE DISTRIBUCIÓN POTENCIAL

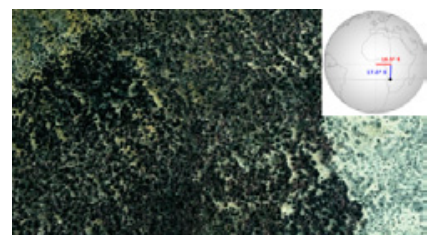

Angola $\left(16^{\circ} 23^{\prime} 8.89^{\prime \prime} \mathrm{S}-17^{\circ} 5^{\prime} 42.95^{\prime \prime} \mathrm{E}\right)$

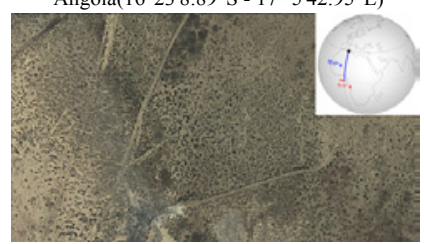

Azerbayan $\left(40^{\circ} 16^{\prime} 14.73^{\prime \prime N}-49^{\circ} 32^{\prime} 20.94 " \mathrm{E}\right)$

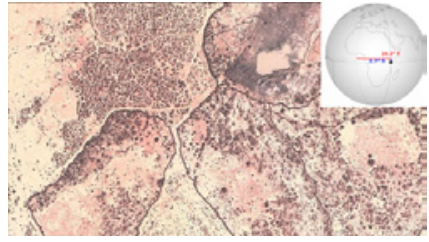

Ethiopia $\left(3^{\circ} 34^{\prime} 23.35^{\prime \prime} \mathrm{N}-39^{\circ} 1^{\prime} 32.67^{\prime \prime} \mathrm{E}\right)$

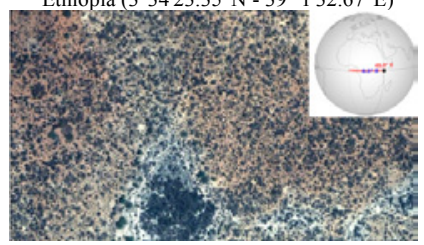

Kenya $\left(0^{\circ} 14^{\prime} 13.20^{\prime \prime} \mathrm{N}-40^{\circ} 26^{\prime} 14.38^{\prime \prime} \mathrm{E}\right)$

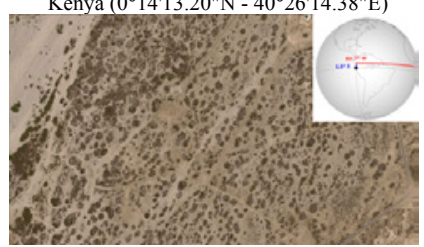

Peru $\left(4^{\circ} 29^{\prime} 8.07^{\prime \prime} \mathrm{S}-81^{\circ} 15^{\prime} 22.35^{\prime \prime} \mathrm{O}\right)$

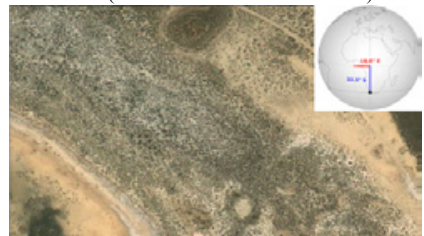

Sudafrica $\left(32^{\circ} 52^{\prime} 29.16^{\prime \prime} \mathrm{S}-18^{\circ} 17^{\prime} 28.66^{\prime \prime} \mathrm{E}\right)$

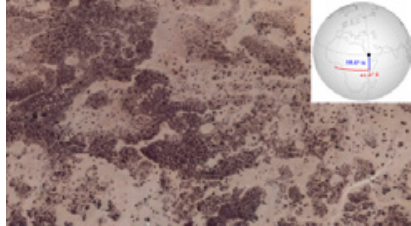

Arabia Saudita $\left(18^{\circ} 39^{\prime} 5.64^{\prime \prime N}-41^{\circ} 25^{\prime} 22.54 " \mathrm{E}\right)$

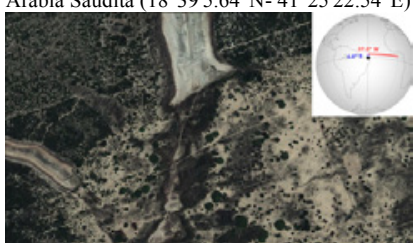

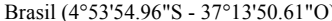
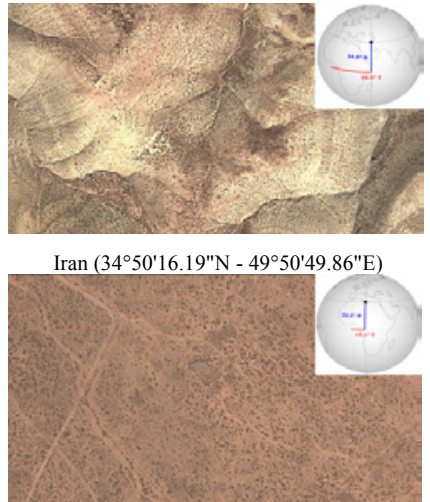

Libia $\left(32^{\circ} 11^{\prime} 40.69^{\prime \prime} \mathrm{N}-15^{\circ} 11^{\prime} 3.06^{\prime \prime} \mathrm{E}\right)$

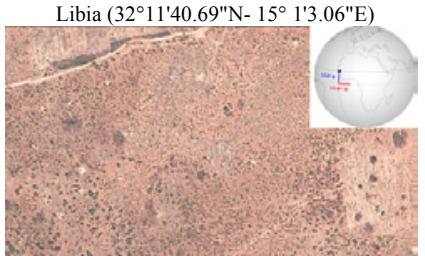

Senegal $\left(13^{\circ} 45^{\prime} 41.86^{\prime \prime} \mathrm{N}-13^{\circ} 44^{\prime} 49.81^{\prime \prime O}\right)$

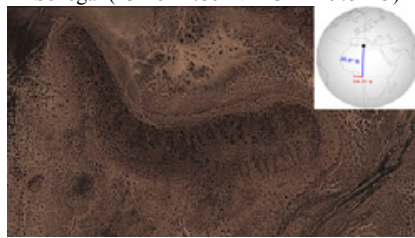

Tunez $\left(35^{\circ} 43^{\prime} 58.20^{\prime \prime} \mathrm{N}-10^{\circ} 45^{\prime} 39.70 " \mathrm{E}\right)$

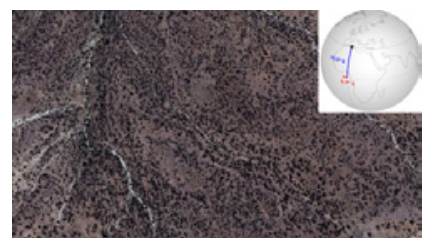

Argelia $\left(35^{\circ} 28^{\prime} 13.71 " \mathrm{~N}-5^{\circ} 37^{\prime} 5.78^{\prime \prime} \mathrm{E}\right)$

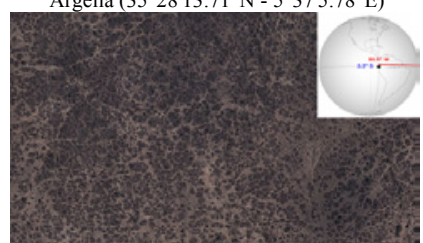

Ecuador $\left(2^{\circ} 11^{\prime} 0.86^{\prime \prime} \mathrm{S}-80^{\circ} 44^{\prime} 15.67^{\prime \prime} \mathrm{O}\right)$

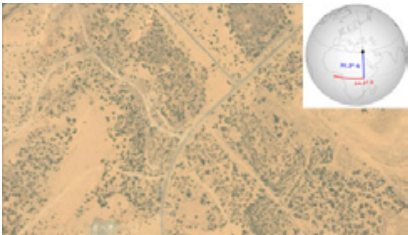

Israel $\left(31^{\circ} 19^{\prime} 34.54^{\prime \prime} \mathrm{N}-34^{\circ} 15^{\prime} 5.38^{\prime \prime} \mathrm{E}\right)$

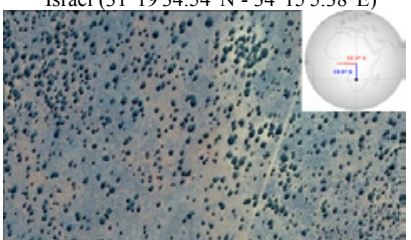

Namibia $\left(23^{\circ} 13^{\prime} 9.51^{\prime \prime} \mathrm{S}-19^{\circ} 3^{\prime} 11.76^{\prime \prime E}\right)$

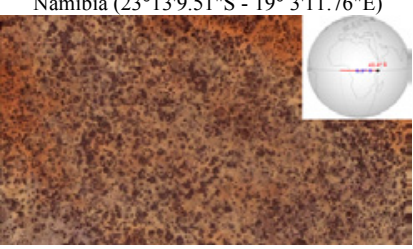

Somalia $\left(0^{\circ} 13^{\prime} 46.89^{\prime \prime} \mathrm{S}-42^{\circ} 30^{\prime} 45.90^{\prime \prime} \mathrm{E}\right)$

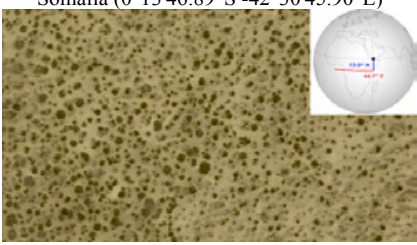

Yemen $\left(12^{\circ} 41^{\prime} 30.52^{\prime \prime N}-44^{\circ} 25^{\prime} 9.07^{\prime \prime} \mathrm{E}\right)$

Fuente: QuickBird, DigitalGlobe distribuido por Google Earth. 


\section{CONCLUSIONES}

El estudio evidencia los rangos de las capas y particularidades que caracterizan a nivel mundial las nuevas ubicaciones en zonas áridas tropicales y subtropicales con patrones leopardo, mejorando la discriminación entre hábitats climáticamente adecuados y no adecuados, en los últimos años a aumentando la disponibilidad y el detalle la información satelital lo que ha permitido mejorar las observaciones y tener mejor detalle aprovechando las disponibilidad de información cartográfica en línea.

Se pudo aislar una relación de ambientes distintivos que claramente conforman patrones y se encuentran en distintos lugares del mundo, más exactamente en distintos continentes, América, África, Europa y Oceanía, la convergencia de estos lugares se debe principalmente a que el ambiente modeló los factores de las formas vegetales de dichas zonas, logrando aislar las características ambientales similares en las especies vegetales, parte de esto se evidenció en dos de las zonas estudiadas en América y Europa, el medio genera los patrones espaciales similares en donde éstas plantas se encuentran, probablemente las especies que estén en otros lugares no analizados arrojen fenómenos de convergencia evolutiva en dichos ambientes similares (Figura 5) o contengan géneros idénticos siendo el caso el de Prosopis en América y Acasia en Australia, África y Europa. (Tabla 1 y 2). La hipótesis de que el ambiente distintivo modela la fenomorfologia de las especies indica que las variables ambientales solamente proporcionan información importante sobre los requisitos del desarrollo del patrón leopardo en una escala regional y que deben haber otros factores más determinantes a nivel local que se desconocen por ahora (Barbier, 2006).

Los patrones tipo leopardo se ubican en su mayoría en zonas exteriores o bordes de grandes desiertos o zonas secas litorales (Sahara, Kalahari, desierto australiano, etc.), principalmente cerca de la zona costera o con algún tipo de influencia marina, una característica especifica que no se ha abordado más a fondo es la posible interacción de estos patrones, relacionado con la presencia de pastos marinos cercanos en las áreas litorales donde hay presencia de patrones leopardo, casos como el de Colombia, España o Australia pueden tener una relación directa en los efectos locales y el aporte de nutrientes principalmente por efectos de los vientos que aportan sedimentos a las áreas marinas cercanas (Hemminga, 1990; Short, 2010).

En los lugares analizados en campo no se ha demostrado que la vegetación sea periódica como sugieren otros autores (Deblauwe, 2008), la variación de vegetación es principalmente el proceso de rebrote o floración de las plantas o el crecimiento temporal de la vegetación por periodos de lluvias, pero el nivel inicial de la vegetación siempre se mantiene a menos que sea perturbado significativamente, también se puede analizar que no solo se limitan a zonas planas pudiéndose presentar en áreas relativamente altas de montañas en todos los continentes, esto es indudable al visualizar los patrones desde un mayor nivel de detalle espacial. Para mejorar la comprensión de cómo interactúan y se conforman los patrones debe haber más investigación de campo que permitan definir las especies vegetales presentes, la variabilidad de nutrientes en el suelo y las características ambientales locales relevantes (vientos, humedad, temperatura, precipitación) además de tener en cuenta la influencia animal. Como en otros estudios concluyen (Aguiar, 1999; Barbier, 2006; Deblauwe, 2008), es probable que el fenómeno de los patrones el algunas zonas que el modelo arrojó, y que actualmente no existe se deba a la supresión de éstos por influencia antrópica, caso probable que se evidenció en zonas de España donde los campos de cultivo e influencia humana podrían haber eliminado patrones antes presentes. 


\section{BIBLIOGRAFÍA}

AGUIAR, M.N.R. y SALA, O.E. (1999): «Patch structure, dynamics and implications for the functioning of arid ecosystems», Trends in Ecology \& Evolution, 14 (7): 273-277.

ARENDT, J. y REZNICK, D. (2008): «Convergence and parallelism reconsidered: what have we learned about the genetics of adaptation?», Trends in Ecology \& Evolution, 23 (1): 26-32.

BARBIER, N., COUTERON, P., LEJOLY, J., DEBLAUWE, V y LEJEUNE, O. (2006): «Self-organized vegetation patterning as a fingerprint of climate and human impact on semi-arid ecosystems», Journal of Ecology, 94 (3): 537-547.

BESTELMEYER, B.T., WARD, J.P.y HAVSTAD, K.M. (2006): «soil-geomorphic heterogeneity governs patchy vegetation dynamics at an arid ecotone», Ecology, 87 (4): 963-973.

BISIGATO, A. J., VILLAGRA, P.E., ARES, J.O. y ROSSI, B.E. (2009): «Vegetation heterogeneity in Monte Desert ecosystems: A multi-scale approach linking patterns and processes», Journal of Arid Environments, 73 (2): 182-191.

BORGOGNO, F., D’ODORICO, P., LAIO, F. y RIDOLFI, L.. (2009): «Mathematical models of vegetation pattern formation in ecohydrology», Reviews of Geophysics, 47 (1): RG1005.

CHENG, Y., STIEGLITZ, M., ENGEL, V. y TURK, G. (2009): Parallel Vegetation Stripe Formation Through Hydrologic Interactions. AGU Fall Meeting Abstracts.

DE PAUW, E., GÖBEL, W. y ADAM, H. (2000): «Agrometeorological aspects of agriculture and forestry in the arid zones», Agricultural and Forest Meteorology, 103 (1): 43-58.

DEBLAUWE, V., BARBIER, N., COUTERON, P., LEJEUNE, O. y BOGAERT, J. (2008): «The global biogeography of semi-arid periodic vegetation patterns», Global Ecology and Biogeography, 17 (6): 715-723.

ELITH, J., PHILLIPS, S.J., HASTIE, T., DUDÍK, M., CHEE, E. y YATES, C.J (2011): «A statistical explanation of MaxEnt for ecologists», Diversity and Distributions, 17 (1): $43-57$.

FRANKLIN, J. (2010): Mapping Species Distributions, Cambridge University Press.

GARCIA-MOYA, E. y MCKELL, C.M. (1970): «Contribution of shrubs to the nitrogen economy of a desert-wash plant community», Ecology, 51:81-88.

HEMMINGA, M.A. y NIEUWENHUIZE, J. (1990): «Seagrass wrack-induced dune formation on a tropical coast (Banc d'Arguin, Mauritania)», Estuarine, Coastal and Shelf Science, 31 (4): 499-502.

HIJMANS, R.J., CAMERON, S.E., PARRA, J.L., JONES, P.G. y JARVIS, A. (2005): «Very high resolution interpolated climate surfaces for global land areas», International Journal of Climatology, 25 (15): 1965-1978.

JARVIS, A., REUTER, H., NELSON, A. y GUEVARA, E. (2008): «Hole-filled SRTM for the globe Version 4.» Disponible en CGIAR-SXI SRTM 90m database: http://srtm. csi. cgiar. org.

JUERGENS, N., OLDELAND, J., HACHFELD, B ., ERB, E. y SCHULTZ, C. (2013): «Ecology and spatial patterns of large-scale vegetation units within the central Namib Desert», Journal of Arid Environments, 93: 59-79.

LEFEVER, R. y TURNER, J.W. (2012): «A quantitative theory of vegetation patterns based on plant structure and the non-local F-KPP equation», Comptes Rendus Mécanique, 340 (11-12): 818-828. 
LOBO, J.M., JIMÉNEZ-VALVERDE, A. y REAL, R. (2008): «AUC: a misleading measure of the performance of predictive distribution models», Global Ecology and Biogeography, 17 (2): 145-151.

MACARTHUR, R.H. (1967): The theory of island biogeography, Princeton University Press.

MEIGS, P. (1953): World Distribution of Arid and Semi-arid Hot Climates. Reviews of Research on Arid Zone Hydrology. Paris, UNESCO.

MERON, E., GILAD, E., VON HARDENBERG, J., SHACHAK, M. y ZARMI, Y. (2004): «Vegetation patterns along a rainfall gradient», Chaos, Solitons \& Fractals, 19 (2): 367-376.

NACHTERGAELE, F. y BATJES, N. (2012): Harmonized world soil database, FAO.

PHILLIPS, S. (2005): «A brief tutorial on Maxent», AT\&T Research.

PHILLIPS, S.J., ANDERSON, R.P. y SCHAPIRE, R.E. (2006): «Maximum entropy modeling of species geographic distributions», Ecological Modelling, 190 (3): 231-259.

PLISCOFF, P. y FUENTES-CASTILLO, T. (2011): «Modelación de la distribución de especies y ecosistemas en el tiempo y en el espacio: una revisión de las nuevas herramientas y enfoques disponibles», Revista de Geografía Norte Grande, 48: 61-79.

RIETKERK, M. y VAN DE KOPPEL, J. (2008): «Regular pattern formation in real ecosystems», Trends in Ecology \& Evolution, 23 (3): 169-175.

SCHLESINGER, W. y PILMANIS, A. (1998): «Plant-soil Interactions in Deserts», Biogeochemistry, 42 (1-2): 169-187.

SHORT, A.D. (2010): «Sediment Transport around Australia-Sources, Mechanisms, Rates, and Barrier Forms», Journal of Coastal Research, 26 (3): 395-402.

SLATYER, R.O. (1959): Methodology of a water balance study conducted on a desert woodland (acacia aneura f. muell.) community in central Australia. Symposium on Plant-Water Relationships in Arid and Semi-arid Conditions. UNESCO. Madrid, UNESCO document: 13 p.

STOCKWELL, D. (1999): «The GARP modelling system: problems and solutions to automated spatial prediction», International Journal of Geographical Information Science, 13(2): 143-158.

STOCKWELL, D.R.B. y NOBLE. I.R. (1992): «Induction of sets of rules from animal distribution data: A robust and informative method of data analysis», Mathematics and Computers in Simulation, 33(5-6): 385-390.

TONGWAY, D.J. y LUDWIG, J.A. (1990): «Vegetation and soil patterning in semi-arid mulga lands of Eastern Australia», Australian Journal of Ecology, 15(1): 23-34.

WARREN, A. (1973): «Some vegetation patterns in the Republic of the Sudan - a discussion», Geoderma, 9 (1): 75-78.

WHITE, D.C. y LEWIS, M.M. (2011): «A new approach to monitoring spatial distribution and dynamics of wetlands and associated flows of Australian Great Artesian Basin springs using QuickBird satellite imagery», Journal of Hydrology, 408 (1-2): 140-152.

WICKENS, G.E. y COLLIER, F.W (1971): «Some vegetation patterns in the Republic of the Sudan», Geoderma 6 (1): 43-59.

WORRALL, G. (1959): «The Butana grass patterns», Journal of Soil Science, 10 (1): 34-53. YETEMEN, O., ISTANBULLUOGLU, E y VIVONI, E.R. (2010): «The implications of geology, soils, and vegetation on landscape morphology: Inferences from semi-arid basins with complex vegetation patterns in Central New Mexico, USA», Geomorphology, 116 (3-4): 246-263. 